\title{
PRODRUG AS A NOVEL APPROACH OF DRUG DELIVERY- A REVIEW
}

\author{
Parajuli Rishi Ram*, Pokhrel Priyanka, Lamichane Shreekrishna, Shrestha Saroj \\ Production Pharmacist, Time Pharmaceutical Pvt. Ltd. \\ *Corresponding Author's Email Positive.rishiram@gmail.com
}

Received 12 April 2015; Review Completed 28 April 2015; Accepted 06 may 2015, Available online 15 May 2015

\begin{abstract}
Prodrugs are bioreversible derivatives of drug molecules that undergo an enzymatic and/or chemical transformation in vivo to release the active parent drug, which can then exert the desired pharmacological effect. Prodrug design is a choice of approach in solving many of the problems like stability, toxicity, solubility, permeability and drug targeting that affect drug discovery and development. Prodrug design is fruitful approach for drug targeting by changing the physiochemical, biopharmaceutical or pharmacokinetic properties of drugs. About $10-14 \%$ of drugs approved worldwide can be classified as prodrugs. The present article takes a review of introduction, classification, applications of prodrug design in various areas of drug development and basic functional groups that are amenable to prodrug design.
\end{abstract}

Keywords: Prodrugs, objectives, classification, application, functional groups, limitation.

\section{INTRODUCTION:}

The term prodrug refers to a pharmacologically inactive compound that is converted to an active drug by a metabolic biotransformation which may occur prior, during and after absorption or at specific target sites within the body. ${ }^{1}$ The concept of "prodrug" was first introduced by Adrian Albert in 1958 to describe compounds that undergo biotransformation prior to eliciting their pharmacological effect i.e. "therapeutic agents that are inactive but can be transformed into one or more active metabolites." The prodrug design approach is also referred to as "Drug Latentiation". 2

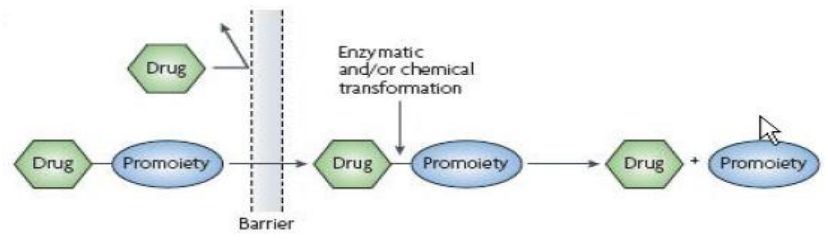

Figure 1: Fate of prodrug approach

Almost all the drugs possess undesirable physiochemical and biological properties such as poor bioavailability, incomplete absorption, adverse effects, first pass metabolism etc. The therapeutic efficacy of these drugs can be easily improved by eliminating the above discussed problems and these problems can be removed by using physical, chemical or biological means. The physical means include introduction of controlled release formulation such as sustained release, prolong release formulation etc. The biological approach is to alter the route of administration which may or may not be acceptable to patient. The third and the last approach is the chemical means which include introduction of prodrug which is best among physical and biological means.

\section{OBJECTIVES OF PRODRUG DESIGN ${ }^{4}$ :}

Pharmaceutical objectives:

$\checkmark$ To improve solubility (e.g., corticosteroids).

$\checkmark$ To improve chemical stability (e.g., dopamine).

$\checkmark$ To improve organoleptic properties (e.g, chloramphenicol palmitate is sparingly soluble prodrug of chloramphenicol, which is practically tasteless due to its low aqueous solubility as well as it is hydrolysed to active chloramphenicol by the action of pancreatic lipase).

$\checkmark \quad$ To decrease irritation and pain.

2. Pharmacokinetic objectives:

$\checkmark \quad$ To improve oral absorption or permeability and thus increase bioavailability(ampicillin,Epinephrine),

$\checkmark$ To decrease first pass metabolism (propanolol).

$\checkmark$ To improve absorption by non oral routes.

$\checkmark$ To provide organ or tissue selective delivery of active agent.

3. Pharmacodynamics objectives:

$\checkmark \quad$ To avoid adverse effects or toxicities.

$\checkmark$ To mask reactive species to improve its therapeutic index.

$\checkmark$ To improve site specificity (i.e., that the site of action of an active drug is rather nonspecific such as anticancer agents). 


\section{CLASSIFICATION OF PRODRUGS: ${ }^{5}$}

Depending upon constitution, lipophilicity, method of bioactivation and catalyst involved; prodrugs are classified into two groups.

A. Carrier - linked prodrugs.

B. Bio precursor prodrug.

\section{Carrier linked prodrug:}

It contains a group that can be easily removed enzymatically (such as ester) to reveal the true drugs. Ideally the group removed is pharmacologically inactive and nontoxic while the connecting bond must be labile for efficient activation in vivo. Carrier linked prodrug consists of the attachment of a carrier group to the active drug to alter its physicochemical properties.

Depending upon the nature of carrirer used it can be classified as:

\section{a) Bipartate Prodrug or Double prodrugs or cascade-latentiated prodrug:}

This prodrug consists of the active drug covalently linked to an inert carrier or transport moiety, generally ester or amide. Such prodrugs have greatly modified lipophilicity due to the attached carrier. The active drug is released by hydrolytic cleavage either chemically or enzymatically. The Prodrug and carrier released after in vivo enzymatical or non-enzymatical attack must be nontoxic.

\section{b) Tripartate Prodrug:}

In this the drug moiety is not directly attached to the carrier moiety.

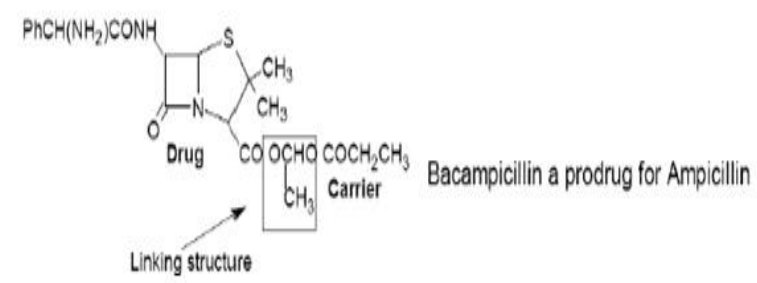

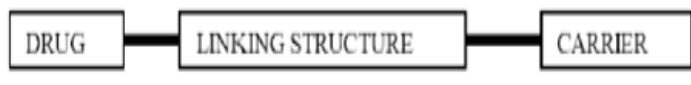

First the drug moiety is attached with the linker and then this linker is attached with carrier.

\section{c. Macromolecular prodrugs:}

Where macromolecules like polysaccharides, dextrans, cyclodextrins, proteins, peptides and polymers are used as carriers.

\section{d. Site- specific prodrugs:}

Where a carrier acts as a transporter of the active drug to a specific targeted site.

\section{e. Mutual prodrug:}

Where the carrier used is another biologically active drug instead of some inert molecule. A mutual prodrug consists of two pharmacologically active agents coupled together so that each acts as a promoiety for the other agent and vice versa. The carrier selected may have the same biological action as that of the parent drug and thus might give synergistic action, or the carrier may have some additional biological action that is lacking in the parent drug, thus ensuring some additional benefit. The carrier may also be a drug that might help to target the parent drug to a specific site or organ or cells or may improve site specificity of a drug. The carrier drug may be used to overcome some side effects of the parent drugs as well.

\section{Bioprecursor/Metabolic precursor:}

This approach does not include any carrier molecule. In this an inactive drug undergoing chemical modification to convert into a compound which itself is active drug or further metabolized into active form which have a desired therapeutic efficacy. The chemical reaction occurs in this process include oxidation or reduction.(e.g. amine to aldehyde to carboxylic acid).
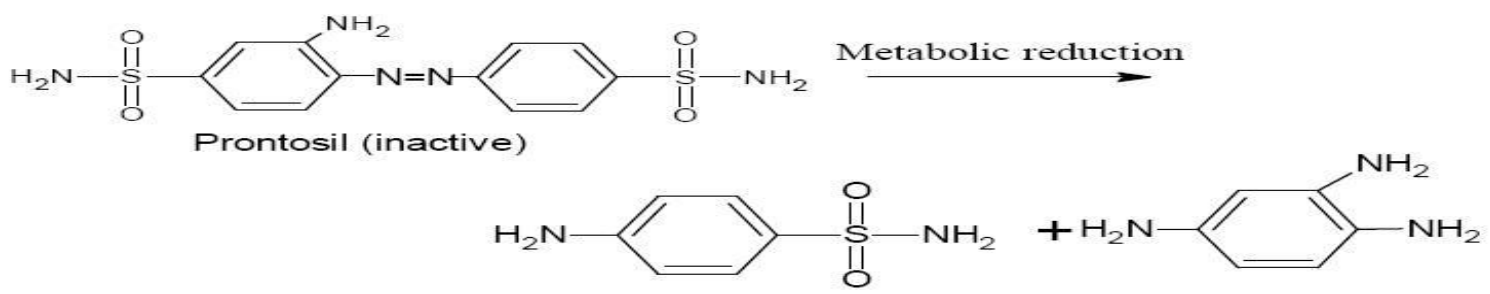

Sulphanilamide ( anitbacterial)
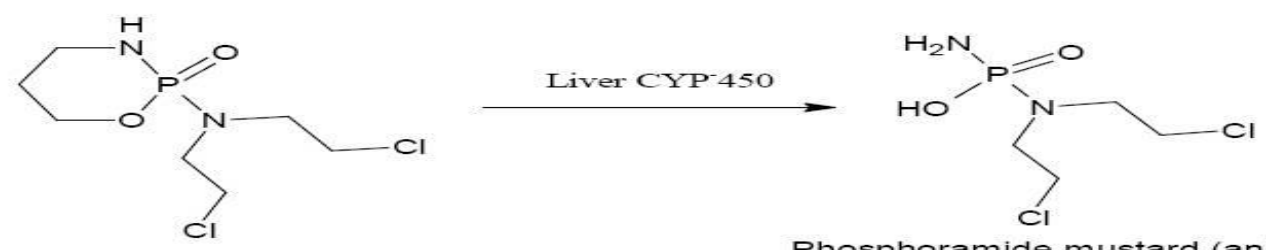

Cyclophosphamide( Inactive)

Phosphoramide mustard (anitneoplasitc)

Examples of bioprecursor prodrugs 


\section{New classification: Based on cellular site of bioactivation:}

Prodrugs can be classified into two major types, based on their cellular sites of bioactivation into the final active drug form with Type I being those that are bioactivated intracellularly (e.g.,anti-viral nucleoside analogs, lipid-lowering statins) and Type II being those that are bioactivated extracellularly, especially in digestive fluids or the systemic circulation (e.g., etoposide phosphate, valganciclovir, fosamprenavir, antibody-, gene- or virus-directed enzyme prodrugs [ADEP/GDEP/VDEP] for chemotherapy or immunotherapy). Both types can be further categorized into Subtypes, i.e. Type IA, IB and Type IIA, IIB and IIC based on whether or not the intracellular bioactivating location is also the site of therapeutic action, or the bioactivation occurs in the GI fluids or systemic circulation. ${ }^{6}$

\section{FUNCTIONAL GROUPS AMENABLE TO PRODRUG DESIGN ${ }^{7}$ :}

Ideally, the design of an appropriate prodrug structure should be considered at the early stages of preclinical development, bearing in mind that prodrugs might alter the tissue distribution, efficacy and the toxicity of the parent drug. Several important factors should be carefully examined when designing a prodrug structure, including

\section{Parent drug:}

Which functional groups are amenable to chemical prodrug derivatization.

\section{Promoiety:}

This should ideally be safe and rapidly excreted from the body. The choice of promoiety should be considered with respect to the disease state, dose and the duration of therapy.

\section{Parent and prodrug:}

The absorption, distribution, metabolism, excretion and pharmacokinetic properties need to be comprehensively understood.

Some of the most common functional groups that are amenable to prodrug design include carboxylic, hydroxyl, amine, phosphate/phosphonate and carbonyl groups. Prodrugs typically produced via the modification of these groups include esters, carbonates, carbamates, amides, phosphates and oximes.

\section{PRODRUG DELIVERY:8,9 \\ INCORPORATED \\ DRUG}

The colloidal drug delivery system works as a controlled and sustained delivery by releasing the encapsulated drug while in circulation or after the recognition by cell, so it is necessary that the delivery system must contain maximum quantity of drug for optimum efficacy.

Liposome: Liposomes consists of lipid bilayer in which between lipid bilayer intervening water molecules are present. The drug is incorporated into either aqueous compartment or in the lipid bilayer as the drug has its @ 2011-15, JDDT. All Rights Reserved physicochemical property. The less hydrophobic drug exhibit low entrapment efficiency and making them more hydrophobic by derivative of fatty acids, improves the entrapment efficiency of delivery system e.g. the tramcinolone palmitate (prodrug) showed 85\% entrapment efficiency as compared to triamcinolone acetonite which has $5 \%$ entrapment efficiency.

Liporotein: Lipoprotein are endogenous transporter of lipids in the circulation, they are nonimmunogenic escapes recognition by reticuloendothelial system. Their structural components are Neo HDL particles consisting of nonpolar triglyceride core surrounded by phospholipids monolayer in which specific apoprotein are imbedded. Since apoprotein are necessary for the recognition of LDL so drug should be into the lipid moiety but most of the drug has not sufficient lipophilic so there is need to prepare lipophilic prodrug.

Emulsion: The oil in water emulsion are used as sustained drug delivery, by passing targeted to macrophages and active targeting by ligand attachment, so in this case the lipophlicity of the drug is necessary to make as oil in water emulsion as sustained delivery system e.g. Esterified phenolic hydroxy derivative of etoposide is used as lipophilic prodrug which is soluble in lipid emulsion in which cholesteryl ester oil used as oil component.

Soild Lipid Nanoparticle: Solid lipid nanoparticle consisted of high melting point triglyceride as the solid core phospholipids coating. Its advantage over the other system is use of natural lipid and incorporation of drug in triglyceride core which may be applicable for prolonged release. For prolonged release it is desirable to incorporate the drugs into triglyceride phase of emulsion e.g. Azidothymidine palmitate ester prodrug incorporation increases as compared to Azidothymidine cleavage.

\section{Methods of Evaluation of Prodrugs ${ }^{10,11}$}

The pharmacokinetics (ADME) of drug is greatly influenced by physicochemical properties such as solubility, lipophilicity, $\mathrm{pH}$, surface area, molecular weight of molecule. Out of this $\mathrm{pH}$, solubility and Lipophilicity are the key factors in determining in vivo behavior of drugs.

Solubility Measurement: The solubility measurement is carried out by placing an excess amount of mutual prodrug in separate vials containing different solvents like $10 \mathrm{ml}$ deionized water, $\mathrm{n}$-hexane, phosphate buffer of different $\mathrm{pH}$ etc and then stirring at $37^{\circ} \mathrm{C}$ for 24 hours. The solutions are centrifuged for $5 \mathrm{~min}$ at 900 RPM and the supernatant is filtered with cellulose acetate membrane filters. The mutual prodrug concentration in each filtrate is determined by suitable analytical technique like HPAE-PAD/UV spectroscopy/HPLC after the appropriate dilution.

Determination of Partition Coefficients: The partition coefficient between water or buffer and n-octanol or cyclohexane is the most widely used measure of chemical compound lipophilicity. Lipophilicity is a major structural factor governing both pharmacokinetics and pharmacodynamics of drugs. The partition 
coefficient of a chemical compound provides ammonium ions. Because the $\mathrm{pH}$ of urine in the bladder is mildly acidic, methenamine is used as a urinary tract antiseptic. To prevent hydrolysis of this prodrug in the acidic environment of the stomach the tablets are enteric coated.

In vitro pH Hydrolysis study: Hydrolysis studies are carried out in aqueous buffer so as to study whether the prodrug hydrolyzes in an aqueous medium and to what extent or not, suggesting the fate of mutual prodrug in the system. The kinetics of hydrolysis is monitored by the increase of free drug concentration with time and the order of the reaction and half-life $\left(\mathrm{t}^{1 / 2}\right)$ are calculated.

\section{APPLICATIONS OF PRODRUGS:}

\section{Anticancer agents:}

Chemotherapeutic agent Paclitaxel was attached to poly (hydroxyl ethyl aspartamide) via a succinic spacer arm by a two-step protocol:synthesis of 2'-O-succinylpaclitaxel; and synthesis of PHEA-2'-O- succinylpaclitaxel. Investigation carried out using murine myeloid cell line showed that the polymeric prodrug maintains partial pharmacological activity of paclitaxel. The conjugate disappeared from the bloodstream much more quickly as compared to both free drug and naked polymer. ${ }^{12}$

\section{In GIT problem: colon targeting:}

For e.g. sulphasalazine which is formed by coupling of diazotized sulphanilamide pyridine with 5-amino salicylic acid. On oral administration intact sulphasalazine reaches the colon. The azo reductase associated with colonic microflora convert sulphasalazin to its constituents entities, the active species 5ASA available for absorption in colon, while precolonic absorption responsible for side effects is reduced. $^{13}$

\section{Immunomodulators:}

Leflunomide is a novel immunomodulatory agent which exhibits a strong anti-inflammatory action. It is potent therapeutic agent in autoimmune diseases, graft rejection, and tumour therapy. It is isoxazole derivative as a prodrug and is completely converted to its active metabolite which blocks the dihydroorotate dehydrogenase, a key enzyme of the pyrimidine de novo synthesis. ${ }^{12}$

\section{Anti-Tubercular agents:}

Ethambutol (EB), isoniazid (INH) and p-amino salicylic acid (PAS) are potent antitubercular agents having various side effects due to formation of toxic metabolites. Mutual prodrugs of EB with PAS (PE), PAS with PAS (PP) and INH with PAS (PI) were synthesized and characterized. In vitro hydrolysis studies in SGF and SIF reveal that these mutual prodrug conjugates do not hydrolyze appreciably and are absorbed unhydrolyzed. In vivo studies showed greater serum concentrations of EB, PAS and INH than their concentrations when given alone and isoniazid concentrations were greater except for PP. Mutual prodrugs PI and PE significantly eliminate the problem of fast metabolism, toxicity and local irritation and reduction of therapeutic doses. ${ }^{14}$

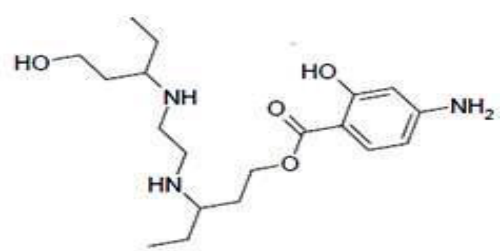

PE

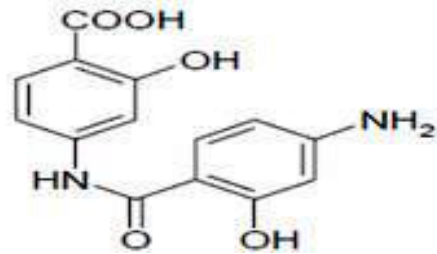

PP

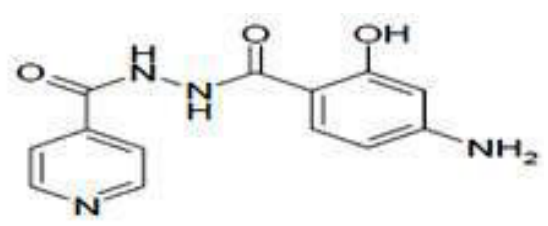

PI

\section{CNS delivery:}

The only prodrug that is used clinically for entering the brain predominantly through LAT1-mediated transport is L-dopa. The neurotransmitter dopamine is not able to cross the BBB due to its hydrophilic nature. However, the conversion of dopamine into its $\alpha$-amino acid, Ldopa, enables the brain to uptake dopamine via LAT1. LDopa is decarboxylated into dopamine by L-amino acid decarboxylase in the brain tissue and also in the peripheral circulation. Although approximately $95 \%$ of L-dopa is metabolized to dopamine in the peripheral tissues, the percentage of remaining L-dopa has been therapeutically enough to apply this approach in clinic practice for more than 30 years. ${ }^{15}$

\section{Ocular delivery:}

The drug pilocarpine was converted to its ester prodrug forms. Pilocarpic acid diester and monoester prodrug solution showed significant biological activity and longer duration of action than pilocarpine. ${ }^{12}$

\section{For treating hypotension:}

L-Threo-3,4-dihydroxyphenylserine (droxidopa) is a norepinephrine (NE) prodrug under development to treat orthostatic hypotension. ${ }^{16}$

\section{Cholesterol-lowering prodrug :}

Simvastatin (SV) is a lactone prodrug which undergoes reversible metabolism. In the hydroxy acid form (SVA) it is a potent inhibitor of HMG-CoA reductase. ${ }^{17}$

\section{LIMITATIONS OF PRODRUG DESIGN: ${ }^{18}$}

As prodrug design has proven highly beneficial in overcoming various undesirable properties of drugs, it can also give rise to a large number of newer difficulties, especially in the assessment of pharmacological, pharmacokinetic, toxicological and clinical properties. 
Problems at the pharmacological level: These compounds cannot be submitted to preliminary in vitro screening tests like binding studies, reuptake of neurotransmitter and enzyme inhibition measurement because bioactivation to their active species is necessary.

Problems at the toxicological level: Formation of toxic metabolite, consumption of vital constituent during prodrug activation process, release of a pharmacokinetic modifier which may cause enzyme induction or alter drug excretion are certain toxicity mechanism of prodrug which is not produced by the parent drugs.

Problems at the pharmacokinetic Studies: The mutual prodrug may not be an ideal substrate for the activating enzymes. Pharmacokinetic studies may lead to numerous misinterpretations. When mutual prodrug and parent molecules are being compared, one must take into account the differences in their respective time courses of action. The maximum activity may appear later for mutual prodrug than for parent compounds, so AUC should be compared as it presents a better criterion for comparison.

Problems at the clinical stage: The predictive value of animal experiments is also questionable. The active doses of two mutual prodrugs of the same parent drugs

\section{REFERENCES:}

1. Tripathi K.D. Essentials of medical pharmacology, $6^{\text {th }}$ edition, 2009, 23

2. Albert A, Nature, 182(B),1958:421.

3. Smith and Williams, Introduction to the Principles of Drug Design and Action, 4th Ed, Taylor and Francis Group, USA: 216- 230.

4. Gennaro, R., Alfanso, W., Remington: The science and practice of pharmacy, Lippincott Williams \& Wilkins, 2003, 20, 1, 913-914.

5. Williams DA. Foye Principles of Medicinal Chemistry, $5^{\text {th }}$ Ed., Lippincott Williams and Wilkins Publishers, Philadelphia, PA.2002.

6. Zawilska JB, Wojcieszak J, Olejniczak AB. Prodrugs: a challenge for the drug development. Pharmacol Rep, 2013; 65(1): $1-14$.

7. Patil SJ and Shirote PJ. Prodrug approach: an effective solution to overcome side-effects. Int J Med Pharm Sci. 2011;1(7):1-13.

8. Ohlanet. Mutual prodrugs- a swot analysis. IJPSR. 2011;2(4):719-729.

9. Longqin HU. Prodrug: Effective solutions for solubility, permeability and challenges Drugs. 2004;7(8).

10. Shargel $A B$ and Andrew BC, Applied Biopharmaceutics \& Pharmacokinetics, 4th Ed.McGraw-Hill Medical Publishing Division, US. 47-66, 129-154. may appear to be same in rats but may be quite different in clinical investigations.

\section{CONCLUSION:}

The prodrug strategy is one of the most promising approaches to enhance the therapeutic efficacy and/or reduce the adverse effects of the pharmacologically active agents via different mechanisms, including increased solubility, stability, improved permeability and bioavailability and tissue-targeted delivery. Hence, prodrugs are becoming a novel approach of the drug discovery paradigm. Despite the remarkable progress made in the field of prodrug design, more studies are clearly needed, especially at early stages of the drug discovery, for prodrugs to achieve the desired state of art and take their place in modern pharmacotherapy.

\section{ACKNOWLEDGMENT:}

The corresponding author expresses deep gratitude to Phr. Prawan Dahal and friends for their co-operation and guidance in searching various articles and journals for completion of this review and also grateful to authors, editors \& publishers of all those articles, journals and books from where the literature for this article has been reviewed \& discussed.

11. Harper NJ and Binns TB. Absorption and Distribution of Drugs. Mdr, India. 1964;103.

12. Shaifali Dubey, Vandana Valecha, Prodrugs: A Review,World Journal of Pharmaceutical Research Vol.3, Issue 7, 277-297.

13. Swarnlata Saraf, Prodrug: A Sustained Chemical Drug Delivery Approach, Pharmaceutical Information Reviews, 5(6), 2007.

14. Rawat J, Jain PK, Ravichandran V, Agrawal RK. Synthesis and evaluation of mutual prodrugs of isoniazid, p-amino salicylic acid and ethambutol. ARKIVOC, 2007; (i):105- 18 .

15. Jarkko R, Krista L, Mikko G, Savolainen J, Prodrugs:Design and clinical applications. AAPS,2008;10(1): 92-102.

16. Holmes C, Whittaker N, Moya JH,Contamination of the norepinephrine prodrug droxidopa by dihydroxy phenylacetaldehyde, Clin. Chem, 2010; 56(5): 832-8.

17. Vickers S, Duncan CA, Chen IW, Metabolic disposition studies on simvastatin, a cholesterol-lowering prodrug. DMD, 1990; 18(2): 2138-45.

18. Longqin Hu, Prodrug:Effective solutions for solubility, permeability \& challengesdrugs,2004;Vol.7, 